\title{
Functional and Structural Aspects of Poplar Cytosolic and Plastidial Type A Methionine Sulfoxide Reductases*
}

Received for publication, May 24, 2006, and in revised form, November 15, 2006 Published, JBC Papers in Press, November 29, 2006, DOI 10.1074/jbc.M605007200

\begin{abstract}
Nicolas Rouhier ${ }^{\ddagger 1,2}$, Brice Kauffmann ${ }^{\S 1}$, Frédérique Tete-Favier ${ }^{\S}$, Pasquale Palladino ${ }^{\S}$, Pierre Gans ", Guy Branlant ", Jean-Pierre Jacquot ${ }^{\ddagger}$, and Sandrine Boschi-Muller

From the ${ }^{\ddagger}$ UMR 1136 INRA-UHP, Nancy Universités, Interactions Arbres Microorganismes, IFR 110, Faculté des Sciences et Techniques, BP 239, 54506 Vandoeuvre Cedex, ${ }^{\S}$ UMR 7036 CNRS-UHP, Nancy Universités, LCM3B, Groupe de Biocristallographie, Faculté des Sciences et Techniques, BP 239, 54506 Vandoeuvre Cedex, "Laboratoire de Résonance Magnétique Nucléaire, Institut de Biologie Structurale, CEA-CNRS-UJF "Jean-Pierre Ebel," 38027 Grenoble Cedex 1, and "UMR 7567 CNRS-UHP, Nancy Universités, Laboratoire MAEM, Groupe Enzymologie Moléculaire, Faculté des Sciences et Techniques, BP 239, 54506 Vandoeuvre Cedex, France
\end{abstract}

The genome of Populus trichocarpa contains five methionine sulfoxide reductase A genes. Here, both cytosolic (cMsrA) and plastidial (pMsrA) poplar MsrAs were analyzed. The two recombinant enzymes are active in the reduction of methionine sulfoxide with either dithiothreitol or poplar thioredoxin as a reductant. In both enzymes, five cysteines, at positions 46,81 , 100, 196, and 202, are conserved. Biochemical and enzymatic analyses of the cysteine-mutated MsrAs support a catalytic mechanism involving three cysteines at positions 46, 196, and 202. $\mathrm{Cys}^{46}$ is the catalytic cysteine, and the two C-terminal cysteines, $\mathrm{Cys}^{196}$ and $\mathrm{Cys}^{202}$, are implicated in the thioredoxin-dependent recycling mechanism. Inspection of the pMsrA $x$-ray three-dimensional structure, which has been determined in this study, strongly suggests that contrary to bacterial and Bos taurus MsrAs, which also contain three essential Cys, the last C-terminal $\mathrm{Cys}^{202}$, but not $\mathrm{Cys}^{196}$, is the first recycling cysteine that forms a disulfide bond with the catalytic Cys ${ }^{46}$. Then $\mathrm{Cys}^{202}$ forms a disulfide bond with the second recycling cysteine Cys ${ }^{196}$ that is preferentially reduced by thioredoxin. In agreement with this assumption, $\mathrm{Cys}^{202}$ is located closer to $\mathrm{Cys}^{46}$ compared with $\mathrm{Cys}^{196}$ and is included in a ${ }^{202} \mathrm{CYG}^{204}$ signature specific for most plant MsrAs. The tyrosine residue corresponds to the one described to be involved in substrate binding in bacterial and $B$. taurus MsrAs. In these MsrAs, the tyrosine residue belongs to a similar signature as found in plant MsrAs but with the first $\mathrm{C}$-terminal cysteine instead of the last C-terminal cysteine.

The production and accumulation of reactive oxygen and nitrogen intermediates, inherent to metabolic processes such as respiration or photosynthesis or to stress conditions, initiate oxidative reactions that affect the biochemical constituents of the cells $(1,2)$. Living organisms use different strategies to pre-

* The costs of publication of this article were defrayed in part by the payment of page charges. This article must therefore be hereby marked "advertisement" in accordance with 18 U.S.C. Section 1734 solely to indicate this fact.

The atomic coordinates and structure factors (code 2J89) have been deposited in the Protein Data Bank, Research Collaboratory for Structural Bioinformatics, Rutgers University, New Brunswick, NJ (http://www.rcsb.org/).

The nucleotide sequence(s) reported in this paper has been submitted to the GenBank TM/EBI Data Bank with accession number(s) AAS46231 and AAS46232.

${ }^{1}$ Both authors contributed equally to this work.

${ }^{2}$ To whom correspondence should be addressed. Tel.: 33-3-83684225; E-mail: nrouhier@scbiol.uhp-nancy.fr. vent oxidative damage and lethal effects that would result from these compounds. Reactive species are trapped and degraded, or modifications that occur anyway are reversed by repair systems, and finally nonrepaired macromolecules can be degraded and removed. Methionine residues of proteins were shown to be one of the preferred targets of oxidation with the formation of methionine sulfoxide (MetSO) $)^{3}$ (3). Enzymes named methionine sulfoxide reductases were found to catalyze the reduction of MetSO back to methionine residues $(4,5)$. The consequences of this side-chain modification are variable and can be partial to protein unfolding $(6,7)$ and modification of biological functions $(8-10)$. Sometimes surface methionine residues can undergo oxidation without much impact on the protein properties, and this modification can be seen as a mechanism to scavenge oxidative species in a detoxification process based on methionine sulfoxide reductase activity (11). Because of its asymmetric sulfur atom, MetSO exists as two stereoisomeric forms, Met- $(S)-\mathrm{SO}$ and Met-(R)-SO. Their reduction back to methionine is catalyzed by two structurally unrelated classes of Msr, MsrAs are specific for Met- $(S)-\mathrm{SO}$, whereas Met- $(R)-\mathrm{SO}$ is the substrate of MsrBs.

MsrAs and MsrBs display no significant sequence identity and have different three-dimensional structures. Only three MsrA x-ray structures from Escherichia coli, Bos taurus, and Mycobacterium tuberculosis and two MsrB structures from Neisseria species have been described so far (12-16). Both classes of Msrs share, for most of them, a similar three-step chemical mechanism, including the following: 1 ) a nucleophilic attack of the catalytic CysA residue on the sulfur atom of the sulfoxide substrate leading to the formation of a sulfenic acid intermediate and to the release of $1 \mathrm{~mol}$ of Met per mol of enzyme; 2) a formation of an intramonomeric disulfide bond between the catalytic CysA and the recycling CysB with a concomitant release of $1 \mathrm{~mol}$ of water; and 3) a reduction of the CysA-CysB methionine sulfoxide reductase disulfide bond by thioredoxin (Trx) (Fig. 1) (17-19). Nevertheless, for MsrAs, at least three subclasses, based on the number and the position of the recycling Cys residues, have been proposed (20). The Neisseria meningitidis and $M$. tuberculosis MsrA represent the first

\footnotetext{
${ }^{3}$ The abbreviations used are: MetSO, methionine sulfoxide; Msr, methionine sulfoxide reductase; DTT, dithiothreitol; Trx, thioredoxin; $\mathrm{TNB}^{-}$, thionitrobenzoate.
} 


\section{Biochemical and Structural Characterization of Plant MsrAs}

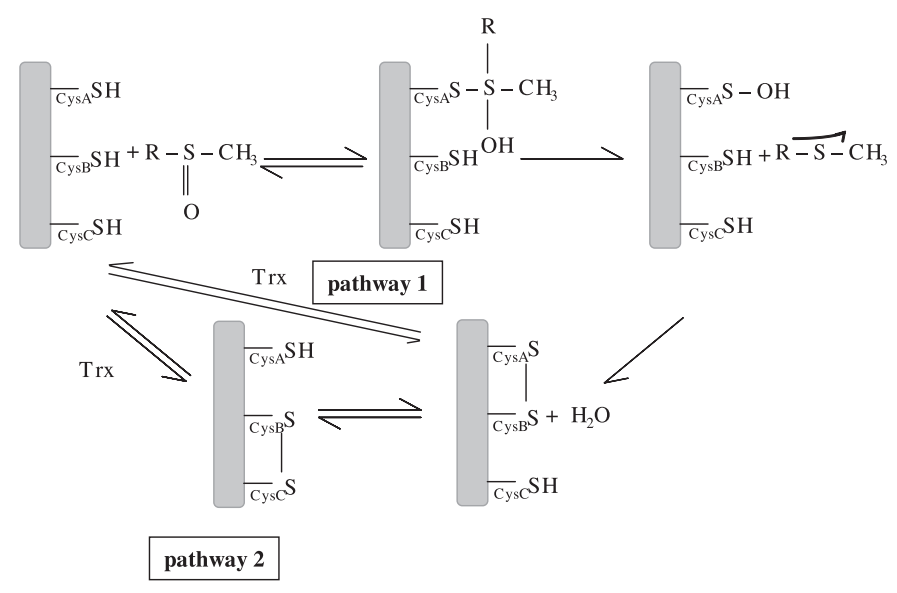

FIGURE 1. Catalytic mechanism of the different subclasses of MsrAs. The mechanism implicates two or three cysteinyl residues, depending on the subclass (CysA, CysB, and possibly CysC) (17). The first step consists of the attack of Cys A onto the sulfur of the methionine sulfoxide, leading to a sulfenic acid on CysA and the concomitant release of the methionine. CysB attacks the sulfenic acid intermediate to form a disulfide bond CysA-CysB. Return of the active site to a fully reduced state proceeds either by direct reduction of the CysA-CysB disulfide bond by Trx (pathway 1) or by two thiol-disulfide exchanges via CysC and Trx (pathway 2), depending on the presence or not of a CysC. For MsrAs possessing a CysC, in the absence of reductant, the first turnover leads to the formation of $1 \mathrm{~mol}$ of Met per mol of enzyme that accumulates under CysB-CysC disulfide bond form. Thus, CysA is free and is able to reduce another MetSO molecule, explaining why the stoichiometry of the reaction is 2 in the absence of reductant.

subclass, characterized by the presence of the recycling CysB in the $\mathrm{C}$-terminal end, and the Bacillus subtilis enzyme represents the second one with the CysB located three amino acids behind CysA. The third subclass, represented by E. coli and B. taurus MsrAs, contains two recycling Cys residues in the C-terminal end and requires the formation not of one but of two successive disulfide bonds. The first one is formed between the catalytic CysA and the recycling CysB. The second one, formed between $\mathrm{CysB}$ and the second recycling cysteine $\mathrm{Cys} C$, is the one preferentially reduced by $\operatorname{Trx}$ in the last step (18). ${ }^{4}$ The denomination of the catalytic cysteines as CysA, $-\mathrm{B}$, and - $\mathrm{C}$ is based on the primary structure order.

Most of the MsrAs studied so far are bacterial or mammalian enzymes. In comparison, little has been done concerning plants. Five MsrA-like genes were identified in Arabidopsis thaliana; one encodes chloroplastic isoforms, and another one is predicted to be targeted to the secretory pathway, and three are cytosolic enzymes $(21,22)$. The expression of the chloroplastic isoform, found mainly in photosynthetic tissues, is strongly induced by illumination of etiolated seedlings and is responsive to various oxidative stress conditions (21-23). Moreover, this plastidial MsrA was also shown to maintain chaperonin activity of a small heat-shock protein Hsp21 by preventing its denaturation and consequently inactivation after methionine oxidation (24). Finally, the expression of cytosolic MsrAs was also shown to respond to various changing conditions as follows: (i) in the dark period of A. thaliana plants growing in short-day conditions (25), (ii) during a pathogen infection by the cauliflower mosaic virus (21), or (iii) during softening of cold-hardened leaves (26). The previous reports

${ }^{4}$ S. Boschi-Muller and G. Branlant, unpublished results. about the plant MsrAs have focused essentially on their expression patterns, but the catalytic mechanism, in particular that related to Trx-dependent recycling process, and the three-dimensional structure of a plant MsrA have not yet been addressed. One of the first methionine sulfoxide reductase activities that was evidenced for a plant enzyme was established for a chloroplast-targeted MsrA from Brassica napus (28).

In this study, the biochemical and catalytic properties of poplar MsrA are presented, in particular those related to the Trx-dependent recycling process. The crystal structure of a poplar MsrA in complex with a mercaptoethanol molecule bound to the catalytic CysA is also reported. Altogether, the data support a Trx-recycling process with formation of a disulfide bond first between the catalytic $\mathrm{Cys}^{46}(\mathrm{CysA})$ and $\mathrm{Cys}^{202}$ (CysC) and then between $\mathrm{Cys}^{202}$ and $\mathrm{Cys}^{196}$ (CysB). This latter disulfide bond was reduced by Trx.

\section{MATERIALS AND METHODS \\ Cloning and Site-directed Mutagenesis}

The open reading frame sequences encoding a cytosolic MsrA (cMsrA) and a plastidial MsrA (pMsrA) (respective GenBank $^{\mathrm{TM}}$ accession numbers AAS46231 and AAS46232) were cloned by PCR into the expression plasmid pET-3d using as templates a root cDNA library of Populus $\times$ interamericana (clone Beaupré) and a leaf cDNA library of Populus tremula $\times$ tremuloides, respectively. Both reactions also contained $P f u$ DNA polymerase (Promega) and the forward and reverse MsrA oligonucleotides described in Table 1. In the pMsrA cloning, a codon for alanine was inserted downstream from the methionine closest to the putative cleavage site and the corresponding $\mathrm{N}$-terminal amino acid sequence starts thus with MANIL. The five cysteines of cMsrA were substituted into serine one by one using either two complementary mutagenic primers per mutation (C46S, C81S, C100S, C196S cMsrA and C46S, C196S pMsrA) (Table 1), using a two-step procedure described previously (28), or a one-step procedure when the mutation is directly inserted in the reverse primers (C202S cMsrA and C202S pMsrA). In addition, various combinations of cysteine substitutions by serine were also introduced in cMsrA (C81S/ C100S; C81S/C100S/C196S; C81S/C100S/C202S; and C81S/ C100S/C196S/C202S cMsrAs). The introduction of the mutation in the cDNA sequence was verified by DNA sequencing.

\section{Expression and Purification of the Recombinant Proteins}

The recombinant plasmids were used to transform the BL21(DE3) E. coli strain, which also contains the helper plasmid pSBET (29). Cultures of 5 liters of a kanamycin-resistant $(50 \mu \mathrm{g} / \mathrm{ml})$ and ampicillin-resistant $(50 \mu \mathrm{g} / \mathrm{ml})$ colony were grown at $37^{\circ} \mathrm{C}$ and induced by $100 \mu \mathrm{M}$ isopropyl 1 -thio- $\beta$-Dgalactopyranoside in the exponential phase. Bacteria were harvested by centrifugation, resuspended in buffer A (30 mM Tris$\mathrm{HCl}, 1 \mathrm{~mm}$ EDTA, $200 \mathrm{~mm} \mathrm{NaCl}$ ) containing $20 \mathrm{~mm}$ DTT, and lysed by sonication. The soluble and insoluble fractions were separated by centrifugation $(16,000 \times g, 30 \mathrm{~min})$. The recombinant wild-type pMsrA was in the soluble fraction and precipitated between 0 and $50 \%$ of ammonium sulfate. All the other recombinant proteins were produced essentially as inclusion bodies with only a small soluble part when cultures were grown at $30{ }^{\circ} \mathrm{C}$ without induction. When needed, the insoluble frac- 


\section{Biochemical and Structural Characterization of Plant MsrAs}

\section{TABLE 1}

\section{Cloning and mutagenic oligonucleotides}

The NcoI and BamHI restriction sites are underlined and the mutagenic bases are in boldface characters.

cMsrA forward
cMsrA reverse
cMsrA C46S forward
cMsrA C46S reverse
cMsrA C81S forward
cMsrA C81S reverse
cMsrA C100S forward
cMsrA C100S reverse
cMsrA C196S forward
cMsrA C196S reverse
cMsrA C202S reverse
pMsrA forward
pMsrA reverse
pMsrA C46S forward
pMsrA C46S reverse
pMsrA C196S forward
pMsrA C196S reverse
pMsrA C202S reverse

pMsrA C202S reverse

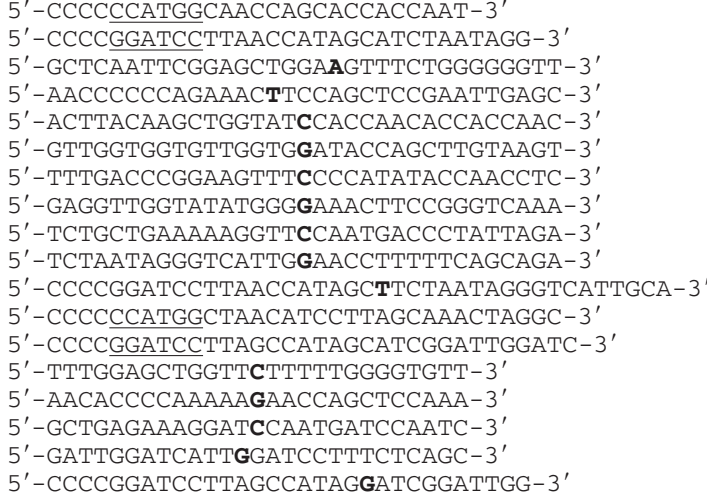

tion was thus resuspended in buffer $\mathrm{A}$ in the presence of $20 \mathrm{~mm}$ DTT and $8 \mathrm{M}$ urea, centrifuged, and then dialyzed against 1 liter of buffer A containing $500 \mathrm{~mm}$ urea for at least $5 \mathrm{~h}$ at $5{ }^{\circ} \mathrm{C}$ (all subsequent steps were realized at that temperature). The extract was centrifuged, and the soluble fraction was dialyzed against 1 liter of buffer $\mathrm{A}$ for $5 \mathrm{~h}$ and finally centrifuged again. The resulting soluble fraction was purified by exclusion size chromatography onto an ACA 44 column equilibrated in buffer A. The fractions of interest were pooled, dialyzed to remove salts, and separated by DEAE-Sephacel chromatography. The recombinant proteins were eluted around $100 \mathrm{~mm} \mathrm{NaCl} \mathrm{using} \mathrm{a}$ linear gradient from 0 to $400 \mathrm{~mm} \mathrm{NaCl}$. The purity of the proteins was assessed using 15\% SDS-PAGE. The protein concentrations were estimated spectrophotometrically using a molar extinction coefficient of $25,700 \mathrm{M}^{-1} \mathrm{~cm}^{-1}$ for cMsrA and pMsrA. The proteins were stored at $-30^{\circ} \mathrm{C}$ in buffer A either in the presence of $14 \mathrm{~mm} \beta$-mercaptoethanol or $25 \mathrm{~mm}$ DTT.

\section{Crystallization, X-ray Data Collection, Structure Determination, and Refinement}

Crystallization of pMsrA was achieved using the hangingdrop vapor-diffusion method in Linbro multiwell tissue culture plates at room temperature. Many crystallization conditions resulted in very thin needles that were not usable for data collection and were impossible to improve. Only one condition described here gave suitable crystals for x-ray crystallography. The purified enzyme was concentrated to $40 \mathrm{mg} / \mathrm{ml}$ in a solution containing $30 \mathrm{~mm}$ Tris- $\mathrm{HCl}, \mathrm{pH}$ 7.0, $14 \mathrm{~mm} \beta$-mercaptoethanol, and 1 mм EDTA. The crystals were grown from 4- $\mu$ l droplets composed of equal volumes of the protein solution and of the precipitant solution (10\% w/v polyethyleneglycol 6000,2 $\mathrm{M} \mathrm{NaCl}$ ) and equilibrated against $700-\mu \mathrm{l}$ reservoirs. Long needles $(1 \mathrm{~mm})$ with a thin triangular cross-section $(0.03 \mathrm{~mm})$ appeared after 6 weeks. Crystals were briefly soaked in a cryoprotectant solution $(10 \% \mathrm{v} / \mathrm{v}$ methylpentanediol mixed with the precipitant solution) and flash-frozen by fast immersion in nitrogen gas stream at $100 \mathrm{~K}$, maintained during the $\mathrm{x}$-ray diffraction experiments performed on beamline BM30A (FIP) at the ESRF.

Crystals belong to space group $\mathrm{P} 3_{1}$ with unit cell parameters $a=b=68.6 \AA, c=40.7 \AA$ and contain one monomer per

\section{TABLE 2}

Statistics of X-ray diffraction data collection for the pMsrA crystals

Values in parentheses refer to data in the highest resolution shell.

\begin{tabular}{ll}
\hline \multicolumn{1}{c}{ Wavelength } & $\mathbf{1 . 0 0 9} \AA$ (ESRF, BM30) \\
\hline Temperature & $100 \mathrm{~K}$ \\
Resolution & $25.0-1.7(1.74-1.70) \AA$ \\
No. of measured reflections & 114,471 \\
No. of independent reflections & 23,121 \\
Completeness & $98.2(83.4) \%$ \\
$R_{\text {sym }}$ & $4.8(21.9) \%$ \\
$\langle\mathrm{I}\rangle /\left\langle\sigma_{I}\right\rangle$ & $8.6(2.5)$ \\
\hline
\end{tabular}

asymmetric unit. Using a wavelength of $1.009 \AA$, one native data set was collected up to a resolution of $1.7 \AA$ and processed using DENZO (30). Further details are given in Table 2.

The structure was solved using the molecular replacement method implemented in Molrep (31) of the CCP4 program suite. The initial model used in Molrep consisted of the core $\left({ }^{41} \mathrm{Gly}-\mathrm{Pro}^{194}\right)$ of the E. coli MsrA structure (Protein Data Bank entry 1FF3). The molecular replacement solution was submitted to the Molrep mode and then to the warpNtrace mode of the Arp/wArp5.1 automatic model building and refinement program (32). It produced a model that contained four polypeptide chains representing 164 amino acids, with $R$ and $R_{\text {free }}$ factors of 20.6 and $25.8 \%$, respectively. Manual corrections (in particular, building of the missing residues) and automatic CNS refinement (33) of the model were then performed in an iterative procedure, until the model fulfilled satisfactory criteria. The final structure corresponds to 183 amino acids among 204 (residues ${ }^{22}$ Pro-Gly ${ }^{204}$ ), 183 water molecules, with $R=19.5 \%$, $R_{\text {free }}=20.1 \%$. Further details are given in Table 3.

\section{Thiol Content Titration}

Known concentrations (generally around $25 \mu \mathrm{M}$ ) of recombinant proteins were reduced with $50 \mathrm{~mm}$ DTT, extensively dialyzed, and then treated or not with $100 \mathrm{~mm}$ L-MetSO for $1 \mathrm{~h}$ at room temperature. The proteins were then precipitated on ice by addition of 1 volume of $20 \%$ trichloroacetic acid for 30 min. The proteins were pelleted by centrifugation and washed twice with $2 \%$ trichloroacetic acid. The pellets were resuspended in $30 \mathrm{~mm}$ Tris- $\mathrm{HCl}, \mathrm{pH}$ 8.0, 1 mm EDTA, and 2\% SDS. The concentrations of the proteins were determined spectrophotometrically at this stage, and then 5,5'-dithiobis(nitroben- 


\section{TABLE 3}

\section{Refinement and model statistics}

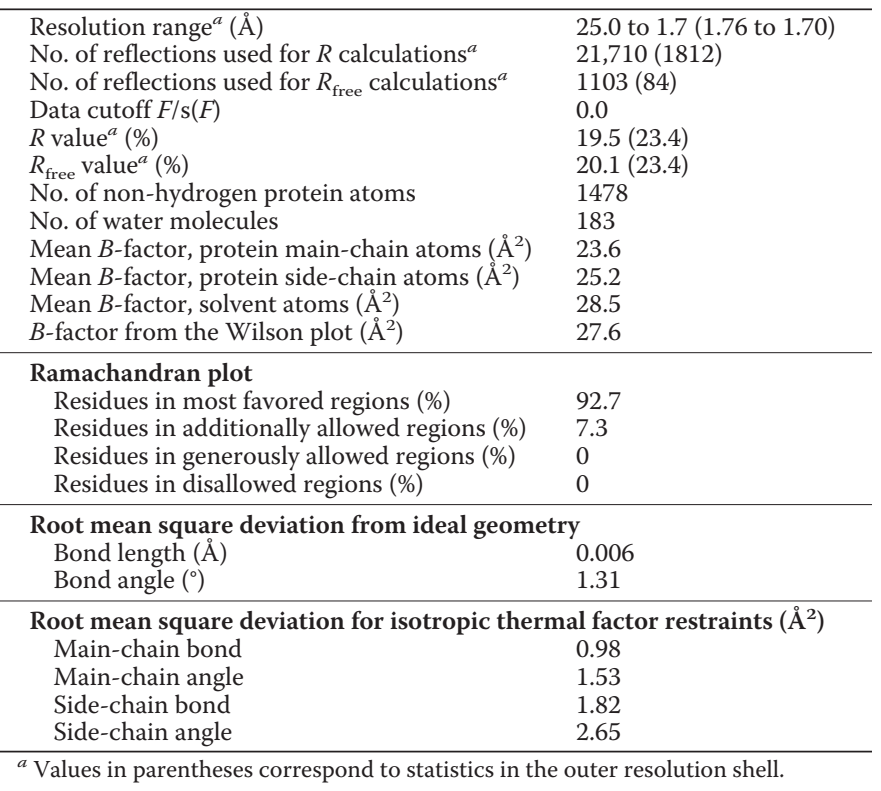

zoic acid) was added to a final concentration of $100 \mu \mathrm{M}$, and the absorbance was read at $412 \mathrm{~nm} 1 \mathrm{~h}$ later. The thiol content was determined using a molar extinction coefficient of $13,600 \mathrm{M}^{-1}$ $\mathrm{cm}^{-1}$ for thionitrobenzoate $\left(\mathrm{TNB}^{-}\right)$.

\section{Characterization of the Sulfenic Acid Intermediate}

The sulfenic acid intermediate was characterized spectrophotometrically by using $\mathrm{TNB}^{-}$under nondenaturing conditions, as described previously (18). Briefly, progress curves of $\mathrm{TNB}^{-}$disappearance were recorded at $412 \mathrm{~nm}$ in $50 \mathrm{~mm}$ Tris$\mathrm{HCl}, \mathrm{pH}$ 8.0, 1 mм EDTA buffer. Enzyme concentrations were 7.35 and $14.7 \mu \mathrm{M}$, and the $\mathrm{TNB}^{-}$concentration was $60 \mu \mathrm{M}$. The amount of $\mathrm{TNB}^{-}$consumed was calculated using an extinction coefficient at $412 \mathrm{~nm}$ of $13,600 \mathrm{M}^{-1} \mathrm{~cm}^{-1}$.

\section{Enzymatic Assays}

NMR Determination of Activity in the Presence of DTT-The catalytic activity was determined by monitoring the reduction of MetSO to Met using DTT as the reducing agent. The concentrations of the substrate and product of the reaction were obtained from the intensity of the resonance signals at 2.65 and 2.15 ppm corresponding to the MetSO methyl resonance and the Met methyl resonance, respectively. The assay conditions were $100 \mathrm{~mm}$ phosphate buffer, $50 \mathrm{~mm}$ DTT, $20 \mathrm{~mm}$ L-Met(RS)-SO at pH 8.5 in 90/10\% $\mathrm{H}_{2} \mathrm{O} / \mathrm{D}_{2} \mathrm{O}$. L-Alanine (10 mM) was used for internal concentration calibration. The enzyme was added directly in the NMR cell, and careful homogenization of the sample was performed just before recording. NMR spectra were recorded with eight scans at $27^{\circ} \mathrm{C}$ every $79 \mathrm{~s}$ on Varian Inova $400 \mathrm{MHz}$ spectrometer equipped with a triple resonance $\left({ }^{1} \mathrm{H},{ }^{13} \mathrm{C}\right.$, and $\left.{ }^{15} \mathrm{~N}\right)$ probe including shielded $z$-gradients. Data were processed using FELIX 97 (Accelrys).

Thioredoxin-dependent Methionine Sulfoxide Reductase Activity-The activity of cMsrA and pMsrA was also measured by following the NADPH oxidation at $340 \mathrm{~nm}$ in the presence of Trx and NADPH Trx reductase system. A 500- $\mu$ l cuvette con- tained 30 mm Tris- $\mathrm{HCl}$, pH 8.0, 1 mм EDTA, $200 \mu \mathrm{M}$ NADPH, $2 \mu \mathrm{M} A$. thaliana NADPH thioredoxin reductase (purified as in Ref. 34), various concentrations of a cytosolic poplar Trx h1, and $100 \mathrm{~mm} \mathrm{~L}$-Met-(RS)-SO. After $1 \mathrm{~min}$ of incubation, MsrA was added to the reaction mixture. Poplar Trx h1 was purified as described previously (35). The reaction was carried out at $30{ }^{\circ} \mathrm{C}$ with a Cary 50 spectrophotometer. The catalytic parameters for Trx and MetSO were determined at saturating concentrations of the other substrate and adjusted using GraFit.

Stoichiometry of Methionine Formation in the Absence of Reductants-The different proteins were reduced by $50 \mathrm{~mm}$ DTT and dialyzed twice against 1 liter of $30 \mathrm{~mm}$ Tris- $\mathrm{HCl}, \mathrm{pH}$ 8.0, 1 mM EDTA. A typical 200- $\mu$ l reaction mixture containing $100-400 \mu \mathrm{M}$ of recombinant proteins and $100 \mathrm{mM} \mathrm{L}-$ Met(RS)-SO was incubated at room temperature for $10 \mathrm{~min}$. After adding $2 \%$ trifluoroacetic acid to stop the reaction, $100 \mu$ l were injected onto a Sephasil C18 column to quantify the concentration of Met formed as described previously (18).

\section{RESULTS AND DISCUSSION}

Genome and Sequence Analysis-Among the five isoforms found in the released genome of Populus trichocarpa, two very close genes (86\% identity) are predicted to be located in plastids and two other (93\% identity) to be cytosolic. Except for the presence of an N-terminal targeting sequence, the four genes are very similar. It is likely that these genes have been actually duplicated two by two. The fifth isoform (EST accession number DT503157) is quite divergent (28-32\% identity) compared with the four other sequences, although it displays the canonical GCFW active site sequence that allows us to classify it as an MsrA, but it does not possess the two C-terminal cysteines (see below). The cDNA sequences of a chloroplastic and a cytosolic isoform, which we call here conveniently pMsrA (plastidial MsrA) and cMsrA (cytosolic MsrA), were isolated by PCR from poplar leaf and root cDNA libraries, respectively. Based on transit peptide prediction programs and amino acid comparisons with homologous proteins from A. thaliana, pMsrA (260 amino acids for the precursor) is predicted to present a 57 amino acid-long $\mathrm{N}$-terminal chloroplastic transit peptide. The size of the mature recombinant pMsrA devoid of the transit peptide produced here (see "Materials and Methods") is 204 amino acids (including the initial methionine and an alanine added for cloning facility). The cmsra open reading frame encodes a protein of 190 amino acids. The additional 14 amino acids of the plastidial form are all located in the N-terminal part of the sequence. The two mature enzymes possess $62 \%$ strict identity at the amino acid level. Fig. 2 displays an amino acid sequence comparison of various plant MsrAs with enzymes from other kingdoms with known catalytic mechanisms or structures.

For the comprehensive analysis of this work, we used the numbering of the recombinant pMsrA both for pMsrA and cMsrA cysteines, although they are not exactly at the same position because of the $\mathrm{N}$-terminal extension in pMsrA. Only the first cysteine at position 46, the catalytic CysA, is conserved among all the sequences presented here. In plants, there are three other strictly conserved cysteines at positions 81,196, and 202, whereas a fourth at position 100 is present in all sequences 
but B. napus. The Cys at position 81 is equivalent to $\mathrm{Cys}^{86}$ of E. coli MsrA, which has been shown to play no role in the catalytic mechanism (18). The C-terminal part of plant MsrAs also contains two cysteines, located in a consensus sequence $\mathrm{K}(\mathrm{G} /$ $\mathrm{V}) \mathrm{C}(\mathrm{I} / \mathrm{N} / \mathrm{K}) \mathrm{DPI}(\mathrm{R} / \mathrm{K}) \mathrm{CYG}$, which is clearly different from those of the E. coli and B. taurus MsrAs. Indeed, in the two latter cases, the C-terminal part is less conserved, with many glycinyl residues around the two recycling cysteines.

Another feature of $E$. coli and B. taurus MsrAs is the presence of a conserved GYC motif around CysB. In few cases, as in $M$. tuberculosis MsrA in which only the CysB is present, an additional residue is inserted before CysB, leading to a GYXC motif. Based on the three-dimensional structure of $M$. tuberculosis MsrA, the tyrosine residue was proposed to participate in the binding of the substrate (14). Interestingly, in plant MsrAs, the GYC motif is neither present near Cys ${ }^{196}$ nor Cys ${ }^{202}$, but the reversed sequence (CYG) is present after the last $\mathrm{C}$-terminal cysteine $\left(\mathrm{Cys}^{202}\right)$.

Thus, based on these $\mathrm{C}$-terminal sequences comparisons, the plant MsrAs could represent a new subclass of MsrA in terms of Trx-recycling process with CysC intervening first to form a disulfide bond with CysA, then followed by formation of a disulfide bond between $\mathrm{CysC}$ and $\mathrm{CysB}$. To validate this hypothesis, the catalytic mechanism of poplar cMsrA and the three-dimensional structure of poplar pMsrA have been investigated.

Methionine Sulfoxide Reductase Activity and Mechanism of the Wild-type Enzymes-The two poplar MsrA isoforms were produced as recombinant proteins and purified to homogeneity. The methionine sulfoxide reductase activity of these two MsrAs was first measured by NMR using DTT as a reductant. As illustrated in Fig. 3, addition of either cMsrA or pMsrA resulted in a rapid decrease of the L-Met- $(R S)$-SO concentration (Fig. $3 A$ ) concomitant with the apparition of Met (Fig. 3B). We have shown previously that pMsrA only reduced L-Met(S)-SO (23). In agreement with the known stereoselectivity of MsrA species, only one-half of the initial L-Met- $(R S)$-SO racemic mixture was reduced after completion of the reaction.

To evaluate further the methionine sulfoxide reductase activity of cMsrA and pMsrA, we used a spectrophotometric test, which coupled the thioredoxin (Trx) system (NADPH/ NADPH thioredoxin reductase/Trx) to NADPH oxidation. Table 4 presents the kinetic parameters of both wild-type enzymes obtained in the presence of a cytosolic poplar Trx called Trx h1. For both MsrAs, the apparent $K_{M}$ values, determined under steady-state conditions for Trx h1 and L-Met$(R S)-\mathrm{SO}$, are around 15 and $300 \mu \mathrm{M}$, respectively. Compared with other biochemically characterized MsrAs, the apparent affinity constant for MetSO is slightly lower (300 $\mu \mathrm{M}$ compared with 600 or $1900 \mu \mathrm{M}$ for N. meningitidis MsrA (36) or E. coli MsrA respectively (18)). Nevertheless, because the rate-limiting step is likely associated with the Trx-dependent recycling process as shown for MsrA from $N$. meningitidis (37) and not to the reductase step, the $K_{M}$ values for MetSO cannot be directly interpreted as representative of a better substrate affinity. It is, however, important to note that the catalytic efficiency, expressed as $k_{\text {cat }} / K_{\text {MetSO }}$, is in the same order for the two poplar MsrAs and for E. coli MsrA.
With the aim to characterize which cysteines are involved in the Trx-recycling mechanism for poplar MsrAs, the stoichiometry of the reductase reaction was measured using high pressure liquid chromatography by following the quantity of methionine formed at a known concentration of reduced MsrA without any other reductant. In parallel, the thiol content of the wild-type MsrAs before and after reduction of L-Met- $(R S)$-SO was estimated (Table 5). The cMsrA and pMsrA exhibit a stoichiometry of nearly $2 \mathrm{~mol}$ of Met by mol of enzyme with a concomitant disappearance of three free thiol groups per monomer (Table 5). These data indicate that three cysteines are involved in the catalytic mechanism, in particular that two of them are implicated in the Trx-dependent regeneration with formation of two successive disulfide bonds. Indeed, based on the scheme in Fig. 1 , if three cysteines are involved, at the end of the reduction of the first MetSO, the catalytic cysteine is free to reduce another MetSO, whereas the recycling two others are under disulfide state. Such a result is similar to that of E. coli MsrA (18).

Role of the Different Cys Residues in the Catalytic MechanismTo investigate the role of the cysteine residues in plant MsrAs, each of the five cysteinyl residues of cMsrA was replaced independently into serine. Among the five monocysteinic mutated MsrAs, only C81S and C100S cMsrAs retained an activity similar to the wild type with DTT or Trx as reductants, indicating that these two cysteines, as expected from their position, are involved neither in MetSO reduction nor in Trx-dependent recycling process. The three other single substitutions affected cMsrA activity whatever the reductant used (data not shown). The C46S cMsrA and C46S pMsrA were both found to be totally inactive with DTT or Trx (Fig. 3 and Table 5), confirming, as expected from its position in the primary sequence, that this Cys residue is the catalytic one. The absence of thiol decrease after MetSO treatment for these two enzymes is also consistent with these results (Table 5).

To further characterize the catalytic mechanism, the cysteines at positions 81 and 100 have been systematically replaced by serine in other mutated MsrAs, leading to triple- and quadruple-substituted cMsrAs called C81S/C100S/C196S, C81S/ C100S/C202S, and C81S/C100S/C196S/C202S cMsrAs. The double-substituted C81S/C100S cMsrA is equivalent to cMsrA in terms of catalytic parameters and stoichiometry of Met formed in the absence of reductant (Tables 4 and 5). The C81S/ C100S/C196S/C202S cMsrA, in which only the catalytic Cys ${ }^{46}$ remains, still displays a MetSO reductase activity (Table 5). The resulting enzyme is not regenerated by $\operatorname{Trx} \mathrm{h} 1$, and its $\mathrm{Cys}^{46}$ is oxidized under sulfenic acid form. Indeed, the decrease in thiol is close to $1 \mathrm{~mol}$ per mol of enzyme and $0.5 \mathrm{~mol}$ of sulfenic acid per mol of enzyme is titrated by $\mathrm{TNB}^{-}$, which is a specific reagent of sulfenic acid derivative.

The stoichiometry of the MetSO reduction by C81S/C100S/ C196S and C81S/C100S/C202S cMsrAs, in the absence of reductant, is 0.43 and 0.82 , respectively (Table 5 ). These results are expected if one of the two cysteines involved in the regeneration of the catalytic $\mathrm{Cys}^{46}$ is removed. This indicates thus that both $\mathrm{Cys}^{196}$ and $\mathrm{Cys}^{202}$ are involved in the regeneration process but does not permit us to conclude the order in which Cys $^{196}$ and Cys ${ }^{202}$ are involved. Both C81S/C100S/C196S and C81S/C100S/C202S cMsrAs were found to be active in the 


\section{Biochemical and Structural Characterization of Plant MsrAs}

pMsra At pMsrA Bn pMsrA Pt pMsrA Ls MsrA Bt

CMsrA Le CMsrA Pi CMsrA Fa pMsra At pMsrA Bn pMsrA Pt pMsrA Ls CMsrA At MsrA Ec MsrA Bt

CMsrA Le CMsrA Pi CMsrA Fa pMsrA At pMsrA Bn pMsrA Pt pMsrA Ls CMsrA At MsrA Ec Msra Bt PilMsra Nm MsrA Mt

CMsrA Le CMsrA Pi cMsrA Fa pMsrA At pMsrA Bn pMsrA Pt pMsrA Ls CMsrA At Msra Ec Msra Bt PilMsrA Nm MsrA Mt

cMsra Le CMsrA Pi CMsra Fa pMsra At pMsrA Bn pMsrA Pt pMsrA Ls CMsra At Msra Ec Msra Bt PilMsra Nm MsrA Mt

CMsra Le CMsrA Pi CMsra Fa pMsrA At pMsrA Bn pMsrA Pt pMsrA Ls CMsrA At MsrA Ec Msra Bt PilMsra Nm MsrA Mt
MOVLVVSPPLIAAASLSKPLNSLS-KAALSFS-RAKPICPFPOT $M L S I V A S P P V I S A V S L S K P L Q S L A-K A A L S L S K R A K P T S P F P K T$ ML QTLSTHLSSTSTSTTTPLLLLSKPFLSPSAKSQLSHS- - - K KF NFPR MFLLRTTTATTTP ... ASLPLPLLSISSHLSLS ... KPSSFPVT $M L S \vee T R R A$

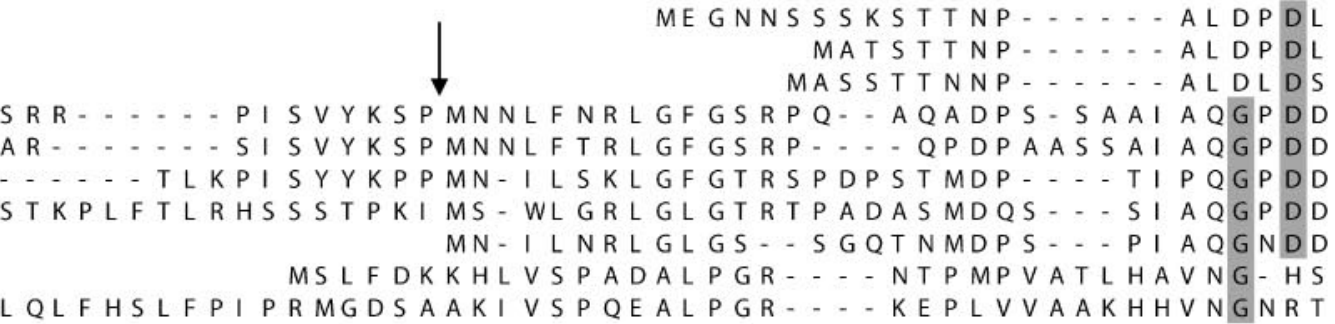

46 (CysA)

DS PDQPGLEFAQFA A GCFWGVELAFQRVGGVVKTEVGYSQGNVHDPNYKLI DQPDNPNHEFAQFGAGCFWGVELAFQRLPGVVKTEVGYSQGHVPDPTYKLV DTPENPGHELAQFASGCF WGSELRFQRVVGVI KTEVGYSQGHVHDPNYRLV DVP. S S GQQFA QFGAGCF WGVELAYQRVPGVTKTEVGYSHGIVHNPSYEDV DVP - SPGQQFA QFGAGCF WGAELAYQRVPGVTKTEVGYSHGFVDNPTYEDV DLP-APGQQFAQFGAGCFWGVELAFQRVPGVTKTEVGYTQGLLHNPTYEDV DI P- APGQQFA QFGAGCF WGVELAFQRVPGVSKTEVGYTQGFLHNPTYNDI DTP- APGNQFA QFGAGCF WGVELAFQR VPGVT QTEAGYTQGTVDNPSYGDV MTN N P DGMEI AI FAMGCF WGVERLFWQLPGVYSTAAGYTGGYTPNPTYREV VEPPPEGT QMAVFGMGCFWGAERKFWTLKGVYSTQVGFAGGYTPNPTYKEV .... MNTRTI YLAGGCF WGLEAYFQRI DGVVDAVSGYANGNTKNPSYEDV -... MT SNQKAI LAGGCFWGLQDLIRNQPGVVSTRVGYSGGNI PNATYRNH

81

100

CS
C T
C S
C T
CS
C S
C T
CS
CS
C S
C S
C S GTTEHAEAI RI QFDPNVCPYSNLLSLFWSRHDPTTLNRQGNDVGKQYRS CTNTTNHVEVVRVQFDPEVCPYTNLLSLFWSRHDPTTLNRQGGDVGTQYRS CS GTTNHSEVVRVQFDPQVCPYSDLLSVFWSRHDPTTLNCQGGDVGTQYRS CT GTTGHNEVVRVQYDPKECSFESLLDVFWNRHDPTTLNRQGGDVGTQYRS CSETTGHNEI VRVQYDPKEVSFESLLDVFWKRHDPTTLNRQGNDVGTRYRS C T G T N H N EV VRVQYDPKECSFDTLIDVLWARHDPTTLNRQGNDVGTQYRS CSGT TNHSEVVRVQYDPKACSFDSLLDCFWERHDPTTLNRQGNDVGTQYRS C S G T T GSEVVRVQYDLNDCTYESLLDLFWSRHDPTTLNRQGNDVGTQYRS CSGDTGHAEAVRIVYDPSVISYEQLLQVFWENHDPAQGMRQGNDHGTQYRS GKT GHAEVVRVVFQPEHISFEELLKVFWENHDPTQGMRQGNDHGSQYRS $S Y R H T G H A E T V K V T Y D A D K L S L D D I L Q Y F F R V V D P T S L N K Q G N D T G T Q Y R S$ GT - - HAEAVEIIFDPTVTDYRTLLEFFFQI HDPTTKDRQGNDRGTSYRS GI Y Y Y NEAQAKLAHESKEAKQLELKDNK- - - VVTEILPAKRFYRAEEYHQ GI Y Y Y NEEQDCLAKKSKEAKQKEFKDKR - - V V TEILPAKRFYRAEEYHQ GI Y Y Y T DE QERI AREAVEKQQK-ILNKR- - - I VTEILPATKFYRAENYHQ GI YFYT DEQEKLAREAMEKQQK-ILNRK- - - I VTEILPATKFYRAENYHQ GI Y Y Y T PEQEKAAKESLERQQK- L L NRK- - - I VTEILPAKKFYRAEEYHQ GI YFYTPEQEKAAI EAKERHQK-KLNRT- - - V VTEILPAKKFYRAEEYHQ GI Y F Y T PEQEKLARESLERHQQ- QMERK- - - I MTEILPAKKFYRAEE H AI Y P L T P E Q A A A R S L ER Q A A MLAADDDRHITTEIANATPFYYAEDDHQ AI Y P TSAEHVGAALKSKEDYQKVLSEHGFG - LITTDIREGQTFYYAEDYHQ GVYYTDPAEKAVIAAALKREQQ-KYQLP - - L L V V K N P P K KFYDAEEYHQ AI F Y F DEQQKRI ALDTI ADVEASGLWPG- - KVVTEVSPAGDFWEAEPEHQ 

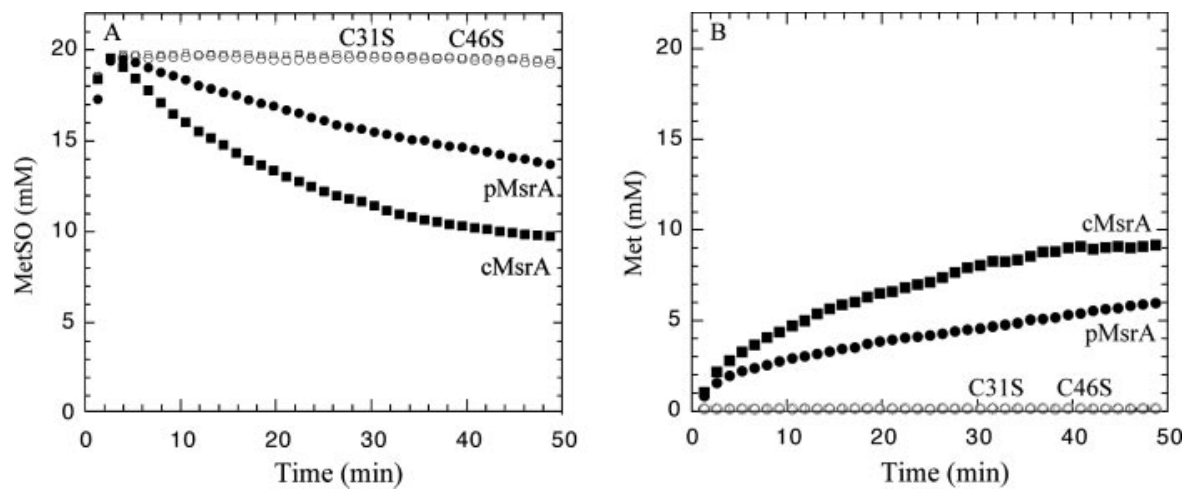

FIGURE 3. MetSO reductive activity of wild-type and mutated cytosolic (cMsrA) and plastidial (pMsrA) poplar sulfoxide methionine reductases. $A$, MetSO concentration evolution as a function of time. $B$, Met concentration evolution as a function of time. pMsrA (filled circles), cMsrA (filled I squares), C46S cMsrA (open squares), C46S pMsrA (open circles). Reactions were recorded at $25^{\circ} \mathrm{C}$ in the presence of $100 \mathrm{~mm}$ phosphate buffer with $50 \mathrm{~mm}$ DTT, $20 \mathrm{~mm}$ L-Met-(RS)-SO at pH 8.5. The enzyme concentrations for cMsrA, pMsrA, C46S cMsrA, and C46S pMsrA were, respectively 4.7, 6.1, 4.7, and $4.9 \mu \mathrm{M}$. MetSO and Met concentrations were determined from the methyl peak intensity of the MetSO at $2.65 \mathrm{ppm}$ and the Met at $2.15 \mathrm{ppm}$.

presence of $\operatorname{Trx}$ (Table 4), and no sulfenic acid was titrated in both triple-mutated cMsrAs with $\mathrm{TNB}^{-}$after MetSO reduction in the absence of reductant. These data show that under the experimental conditions used, i.e. $10 \mathrm{~min}$ of incubation, a disulfide bond is formed whatever the nature of the substituted recycling Cys. Based on the catalytic mechanism of the E. coli and of B. taurus MsrAs, which both support the successive involvement of the two recycling Cys, these results are rather unexpected (see Fig. 1). Indeed, a titration of one sulfenic acid was expected for the substitution of the first recycling cysteine, whereas no sulfenic titration was expected for the substitution of the second recycling cysteine because of formation of a disulfide bond in this latter case. Other unexplained data come from the thiol titrations. Indeed, titration of free thiol indicates loss of 0.7 and 0.8 thiols after MetSO reduction in the absence of reductant for the C81S/C100S/C196S and C81S/C100S/C202S cMsrAs, respectively (Table 5), whereas a loss of either 1 or 2 was expected. Increasing the incubation time or concentrations of the substrate or enzyme did not change significantly the data on thiol titration. These results remain to be explained and could be related to the observed instability of these substituted cMsrAs.

When looking at the Trx-dependent activity, the C81S/ C100S/C196S and C81S/C100S/C202S cMsrAs displayed modified catalytic parameters compared with those of C81S/ C100S cMsrA (Table 4). First, the $K_{M}$ values of C81S/C100S/ C196S and C81S/C100S/C202S cMsrAs for Trx h1 are increased around 12- and 6-fold compared with that of C81S/ C100S cMsrA and cMsrA, respectively. On the other hand, the $k_{\text {cat }}$ value of the two C-terminal cysteine-mutated MsrAs is decreased by 3- and 9-fold, respectively, with C81S/C100S/ C202S cMsrA being the more affected. In terms of catalytic efficiency $\left(k_{\mathrm{cat}} / K_{\mathrm{Trx}}\right)$, a decrease of 36-fold for C81S/C100S/C196S cMsrA and of 107-fold for C81S/ C100S/C202S is observed compared with the C81S/C100S cMsrA. Together, these results indicate that the disulfide bond between $\mathrm{Cys}^{46}$ and $\mathrm{Cys}^{202}$ is the more efficiently reduced by $\operatorname{Trx}$ in terms of $k_{\text {cat }}$. The fact that, because of a lower $K_{M}$ value for $\operatorname{Trx}$, the $k_{\mathrm{cat}} / K_{\mathrm{Trx}}$ values are significantly higher for $\mathrm{cMsrA}$ and C81S/C100S cMsrA compared with that of C81S/C100S/C196S and C81S/C100S/C202S cMsrAs supports the intervention of Trx on the disulfide $\mathrm{Cys}^{196} / \mathrm{Cys}^{202}$ bond and not on $\mathrm{Cys}^{46} / \mathrm{Cys}^{196}$ or $\mathrm{Cys}^{46} /$ $\mathrm{Cys}^{202}$ bonds. Indeed, a lower $K_{M}$ value for $\operatorname{Trx}$ necessitates less $\operatorname{Trx}$ in vivo to regenerate the catalytic CysA. In terms of evolution, this is certainly an advantage that has already been shown for E. coli MsrA. ${ }^{4}$

Thus, the results presented here clearly show the following: (i) $\mathrm{Cys}^{46}$ is the catalytic CysA, and (ii) the recycling process implicates $\mathrm{Cys}^{196}$ and $\mathrm{Cys}^{202}$ but does not discriminate from the order in which $\mathrm{Cys}^{196}$ and $\mathrm{Cys}^{202}$ are involved. To answer this question, it would be necessary to determine the rate of the disulfide bond formation in each triple-substituted cMsrA in which only one recycling cysteine is present. These rates are likely very different depending on the recycling cysteine and its position relative to the catalytic one. Unfortunately, the nonreliable thiol titration on both mutated cMsrAs cannot permit us to attain these rates experimentally. Thus, to get further insights into the Trx-dependent recycling process of plant MsrA, the determination of the three-dimensional structure of pMsrA has been undertaken.

Overall Structure of $p M s r A-$ Crystallization trials were performed for both chloroplastic and cytosolic MsrA in the reduced and oxidized forms, but crystals suitable for $\mathrm{x}$-ray analysis were obtained only for pMsrA in the reduced form. The trigonal crystals (space group $\mathrm{P} 3_{1}$ ) of pMsrA contain one molecule per asymmetric unit and $45 \%$ of solvent (Tables 2 and 3 ). The structure was solved by molecular replacement and refined up to a resolution of $1.7 \AA$ with final $R$ and $R_{\text {free }}$ factors of 19.5 and $20.1 \%$, respectively. No electron density was observed upstream from $\mathrm{Pro}^{22}$ in the $\mathrm{N}$-terminal part. The missing residues ${ }^{1}$ Met-Asp ${ }^{21}$ are very disordered and probably situated in the large solvent cavity observed in the crystal packing interface. The catalytic $\mathrm{Cys}^{46}$ (CysA) and the recycling Cys ${ }^{196}$ (CysB) and $\mathrm{Cys}^{202}$ (CysC) but also $\mathrm{Cys}^{81}$ clearly show extra densities on

FIGURE 2. Amino acid comparison of the two poplar MsrAs with plant MsrA sequences and biochemically or structurally characterized MsrAs. The sequences were compared using ClustalW. The accession numbers (GenBank ${ }^{\mathrm{TM}}$ or MATDB) are as follow: P. tremula $\times$ tremuloides pMsrA (Pt pMsrA), AY530805; A. thaliana cMsrA (At CMsrA), At5g61640; A. thaliana pMsrA (At pMsrA), At4g25130; B. napus pMsrA (Bn pMsrA), P54151; P. X interamericana cMsrA (Pi cMsrA), AY530804; Lactuca sativa (Ls pMsrA), Q9SEC2; F. ananassa (Fa cMsrA), P54153; Lycopersicum esculentum (Le CMsrA), P54153; E. coli MsrA (Ec MsrA), NP 418640; B. taurus MsrA (Bt MsrA), P54149; M. tuberculosis (Mt MsrA), NP_334555; and N. meningitidis (Nm PilB MsrA), E82024. The cysteine numbering of cMsrA and pMsrA is indicated, with CysA corresponding to Cys ${ }^{46}$, CysB to Cys ${ }^{196}$, and CysC to Cys ${ }^{202}$. The black arrow indicates the putative peptide cleavage site. In white on black are indicated the cysteinyl residues and the GYC, GYXC, or CYG signatures. In black on gray are presented the other very conserved or fully conserved residues. 


\section{Biochemical and Structural Characterization of Plant MsrAs}

\section{TABLE 4}

Catalytic parameters of wild-type pMsrA and cMsrA and mutated cMsrAs

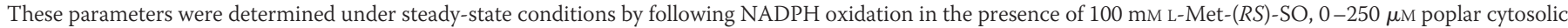

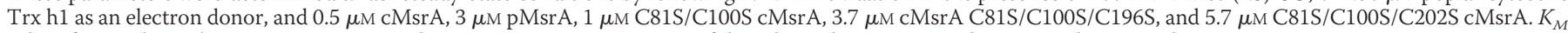
values for Trx h1 and MetSO were measured at saturating concentrations of the other substrate. ND indicates not determined.

\begin{tabular}{|c|c|c|c|c|c|}
\hline & $K_{\text {Trx }}$ & $K_{\text {MetSo }}$ & $k_{\text {cat }}$ & $k_{\text {cat }} / K_{\text {MetSO }}$ & $k_{\mathrm{cat}} / K_{\mathrm{Trx}}$ \\
\hline & $\mu M$ & $\mu M$ & $s^{-1}$ & $\times 10^{3} M^{-1} s^{-1}$ & $\times 10^{3} M^{-1} s^{-1}$ \\
\hline $\mathrm{EcMsrA}^{a}$ & $10 \pm 2$ & 1900 & 3.7 & $2.0 . \pm 0.5$ & $370 \pm 70$ \\
\hline pMsrA & $18 \pm 8$ & $240 \pm 30$ & $0.30 \pm 0.03$ & $1.2 \pm 0.3$ & $17 \pm 6$ \\
\hline cMsrA & $15 \pm 3$ & $380 \pm 100$ & $1.20 \pm 0.10$ & $3.1 \pm 0.6$ & $80 \pm 19$ \\
\hline C81S/C100S cMsrA & $8 \pm 1$ & ND & $0.60 \pm 0.04$ & ND & $75 \pm 9$ \\
\hline C81S/C100S/C196S cMsrA & $94 \pm 15$ & ND & $0.20 \pm 0.02$ & ND & $2.1 \pm 0.5$ \\
\hline C81S/C100S/C202S cMsrA & $103 \pm 17$ & ND & $0.07 \pm 0.01$ & ND & $0.7 \pm 0.1$ \\
\hline
\end{tabular}

${ }^{a}$ Results were obtained with E. coli MsrA, from Boschi Muller et al. (18).

\section{TABLE 5}

Stoichiometry of Met formed in the absence of reductant and decrease in thiol content after treatment or not with L-Met-(RS)-SO for wild-type pMsrA and cMsrA and mutated cMsrAs

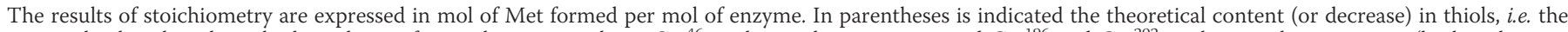

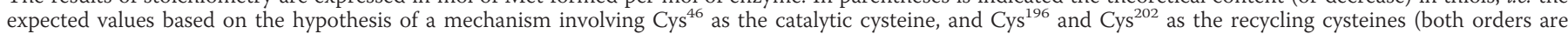
considered).

\begin{tabular}{|c|c|c|c|c|}
\hline & Stoichiometry & $\begin{array}{l}\text { Number of free thiols } \\
\text { without L-Met- }(R S)-\text { SO }\end{array}$ & $\begin{array}{l}\text { Number of free thiols } \\
\text { with L-Met- }(R S) \text {-SO }\end{array}$ & Decrease in free thiols \\
\hline pMsrA & 1.8 & $4.3 \pm 0.4(5)$ & $1.3 \pm 0.1(2)$ & $3(3)$ \\
\hline cMsrA & 1.7 & $4.4 \pm 0.4(5)$ & $1.1 \pm 0.1(2)$ & $3.3(3)$ \\
\hline C46S cMsrA & 0 & $3.9 \pm 0.4(4)$ & $3.8 \pm 0.3(4)$ & $0.1(0)$ \\
\hline C81S/C100S cMsrA & 1.5 & $2.7 \pm 0.3(3)$ & $0.10 \pm 0.02(0)$ & $2.6(3)$ \\
\hline C81S/C100S/C196S cMsrA & 0.43 & $1.5 \pm 0.1(2)$ & $0.8 \pm 0.1(0$ or 1$)$ & $0.7(1$ or 2$)$ \\
\hline C81S/C100S/C202S cMsrA & 0.82 & $1.6 \pm 0.1(2)$ & $0.8 \pm 0.1(0$ or 1$)$ & $0.8(1$ or 2$)$ \\
\hline C81S/C100S/C196S/C202S cMsrA & 0.80 & $1.0 \pm 0.1(1)$ & $0.10 \pm 0.02(0)$ & $0.9(1)$ \\
\hline
\end{tabular}

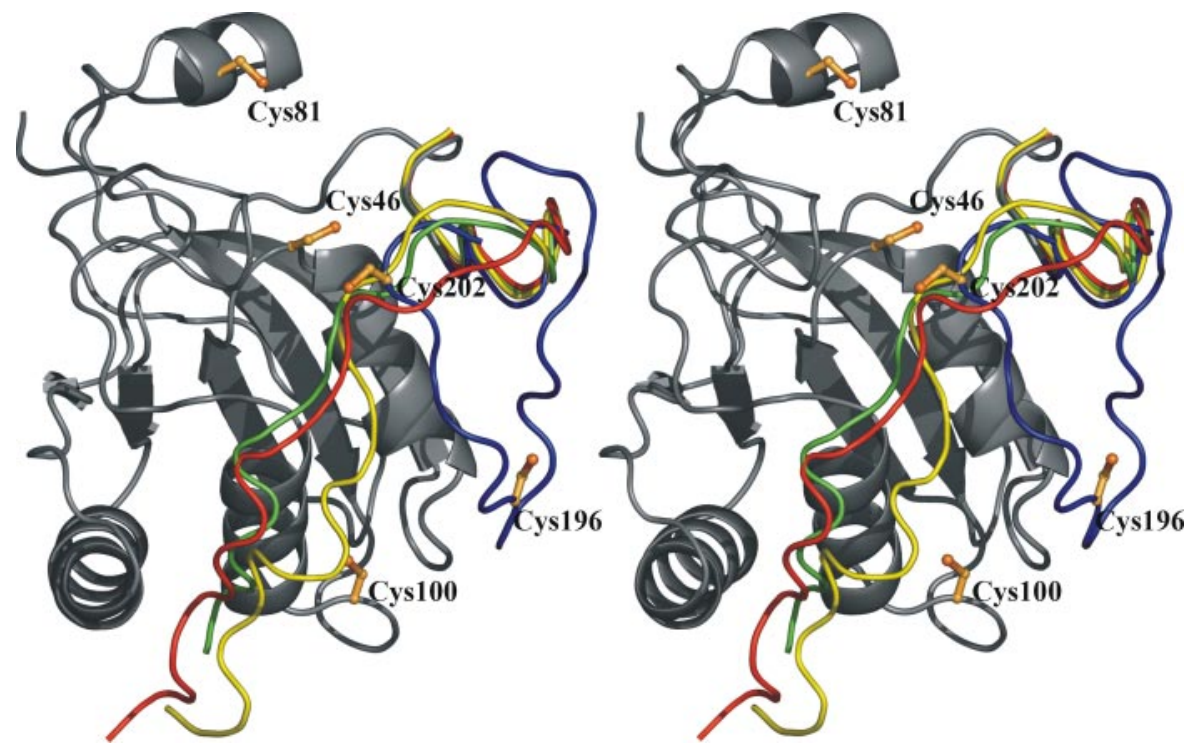

FIGURE 4. Stereoview of the pMsrA monomer structure. The core enzyme is shown in gray and the C-terminal part in blue. The C-terminal ends of the E. coli, B. taurus, and M. tuberculosis MsrA superimposed to the poplar model are shown in red, green, and yellow, respectively. The five cysteine residues present in the pMsrA structure are drawn in ball-and-stick. This figure was generated using Pymol (The PyMOL Molecular Graphics System is available on line).
On the contrary, major differences concern the $\mathrm{N}$ - and C-terminal ends of the poplar enzyme. Because the $C$-terminal end contains two of the three cysteines involved in the catalytic mechanism (Cys ${ }^{196}$ and $\mathrm{Cys}^{202}$ ), it is of high interest to compare it with other MsrAs.

In E. coli, B. taurus, and M. tuberculosis MsrAs, the C-terminal ends, starting from structurally equivalent positions 192, 212, and 147, respectively, are observed as long extended coils without tertiary organization, which lean against the core of the domain and run roughly in the same direction, at the border of the active site. On the contrary, in the pMsrA model, the ${ }^{182}$ LysGly $^{204}$ C-terminal end is observed for the first time in a totally new stabilized conformation (Fig. 4). The polypeptide chain turns by $90^{\circ}$ with their sulfur atoms that were unambiguously modeled as bonded $\beta$-mercaptoethanol, present in the crystallization solution. Surprisingly, Cys ${ }^{100}$, the most solvent-exposed cysteine, was observed in its reduced form.

As expected from the sequence identity, the poplar pMsrA model displays the same overall fold than the E. coli, B. taurus, and M. tuberculosis MsrAs (respective Protein Data Bank entries 1FF3 (12), 1FVA, 1FVG (13), and 1NWA (14)). C- $\alpha$ atoms corresponding to the cores of these enzymes can be superimposed with root mean square distances of about $0.8 \AA$. respect to the previously described $\mathrm{C}$-terminal ends, so that it starts to run parallel to the first $\alpha$-helix (named H1) of the central core of the structure and then, after a turn, antiparallel to it. It results in a packed conformation, as opposed to the extended conformation previously observed for the C-terminal ends of other MsrA structures. Numerous hydrogen bonds are observed within this C-terminal part and between the C-terminal part and the core of the enzyme. Three amino acid residues, only and strictly conserved in plant MsrA sequences, seem to play a key role in the global architecture of the C-terminal part 


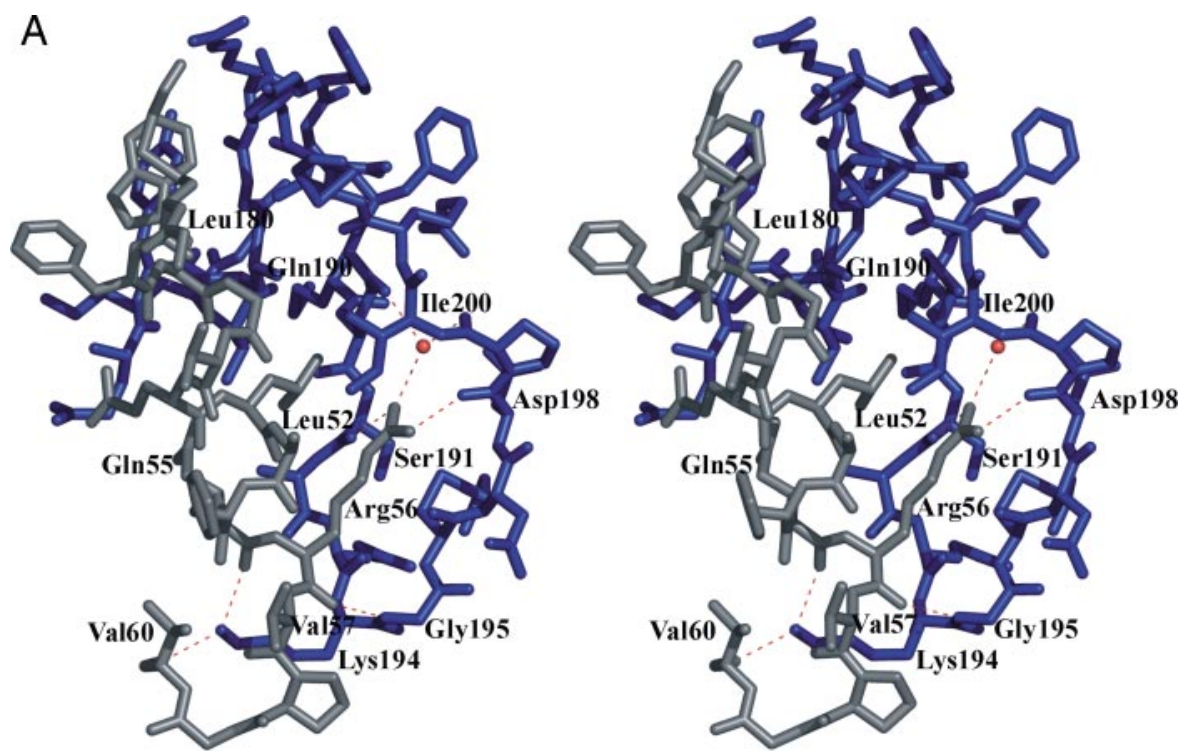

Interestingly, the KGG sequence is also conserved in other plant MsrAs. The lack of the proline and the presence of two glycine residues in pMsrA strongly modify the torsion constraints in this region.

The conformation of the C-terminal end observed in the poplar pMsrA structure does not seem to arise from the crystal form studied in this $\mathrm{x}$-ray analysis. Indeed, even if this part of the structure faces two monomers in the crystal lattice, the few observed interactions with the neighboring partners could not account for a misfolding of the 23 C-terminal residues, especially because this new conformation of the $\mathrm{C}$-terminal end features several
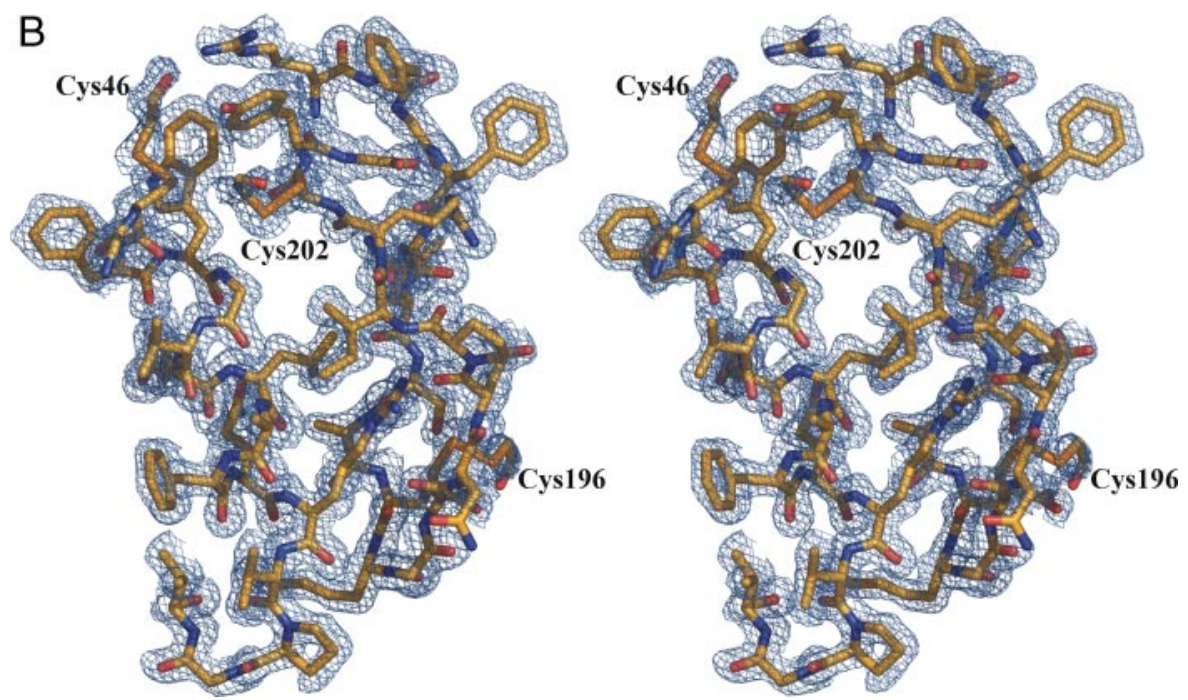

FIGURE 5. Stereoviews of the pMsrA C-terminal end. $A$, the C-terminal end of pMsrA is shown in blue, and the core of the enzyme is displayed in gray. The hydrogen bonds shared between the C-terminal domain and the core of pMsrA are shown as dashed lines. The red sphere corresponds to a water molecule. $B$, the $2 F_{o}-F_{c}$ electronic density (contour level: $1.2 \sigma$ ) superimposed on the pMsrA C-terminal part. intramolecular interactions that involve residues conserved exclusively in plant sequences. Analysis of the active site, developed below, reinforces this assumption.

Modification of the Catalytic Cysteine and Architecture of the Active Site-The side chain of the catalytic cysteine Cys ${ }^{46}$ (CysA) conserves the same position in the poplar pMsrA structure with respect to the other known MsrA x-ray models. The $\beta$-mercaptoethanol molecule bonded to $\mathrm{Cys}^{46}$ occupies a site situated within the active site. The modification of CysA has been observed in other MsrA structures. Indeed, in E. coli MsrA, a dimethylarsenate group resulting from the crystallization medium is bonded to Cys $^{51}$ (CysA) (12). In the B. taurus

(Fig. 5). First, the $\mathrm{N}-\eta 1$ and $\mathrm{N}-\eta 2$ atoms of $\mathrm{Arg}^{56}$, located at the end of the $\alpha$-helix H1, interact with the carbonyl groups of Asp $^{198}$ and Ser ${ }^{191}$, respectively, whereas its carbonyl group is hydrogen-bonded with the $\mathrm{NH}$ group of Gly ${ }^{195}$. Second, the $\mathrm{N}-\zeta$ atom of Lys ${ }^{194}$ is hydrogen-bonded with the carbonyl groups of $\mathrm{Gln}^{55}, \mathrm{Val}^{57}$, and $\mathrm{Val}^{60}$ situated in the loop connecting the $\alpha$-helix $\mathrm{H} 1$ with the following $\beta$-strand. Finally, $\operatorname{Gln}^{190}$ contributes to the stabilization of the $\mathrm{C}$-terminal end via an interaction between its elongated side chain and the main chain of $\mathrm{Arg}^{201}$. Furthermore, a water molecule that interacts with Gln ${ }^{190}, \mathrm{Asp}^{198}$, Pro ${ }^{199}$, and $\mathrm{Arg}^{56}$ further stabilizes the packed conformation of the $\mathrm{C}$-terminal end. When superimposing the structures of E.coli, B. taurus, M. tuberculosis, and poplar pMsrA, the main chain of the latter enzyme starts to deviate from the others at a position that can be considered as a hinge region. It consists of the sequence ${ }^{182} \mathrm{KGG}^{184}$ in poplar MsrA, whereas it is ${ }^{192} \mathrm{KNP}^{194}$ in the $E$. coli MsrA, ${ }^{212} \mathrm{KDP}^{214}$ in the $B$. taurus sequence, and ${ }^{147} \mathrm{RYP}^{149}$ in the $M$. tuberculosis enzyme. structure, a bound dithiothreitol molecule is observed on $\mathrm{Cys}^{72}$ (CysA) (13). Furthermore, in the M. tuberculosis MsrA structure, a crystal contact fortuitously places the methionine side chain of a neighboring monomer in the active site of the enzyme (14). These different complexes have been proposed to mimic either a Michaelis complex or a transient intermediate.

The position of the $\beta$-mercaptoethanol molecule observed on CysA in the pMsrA structure supports the proposals made for the recognition and stabilization of the substrate $(13,14)$. Indeed, the sulfur atom of the $\beta$-mercaptoethanol molecule bonded to $\mathrm{Cys}^{46}$ occupies the same position as the arsenic atom that mimic the sulfur atom of methionine sulfoxide in E. coli MsrA. The entire $\beta$-mercaptoethanol molecule elongates in the same direction as the methionine side chain in the M. tuberculosis active site (14) and as the dithiothreitol molecule present in the B. taurus MsrA (13). Consequently, Asp ${ }^{124}$, which is conserved in most MsrA sequences, forms a hydrogen bond with the hydroxyl group of the $\beta$-mercaptoethanol molecule, similar 
A
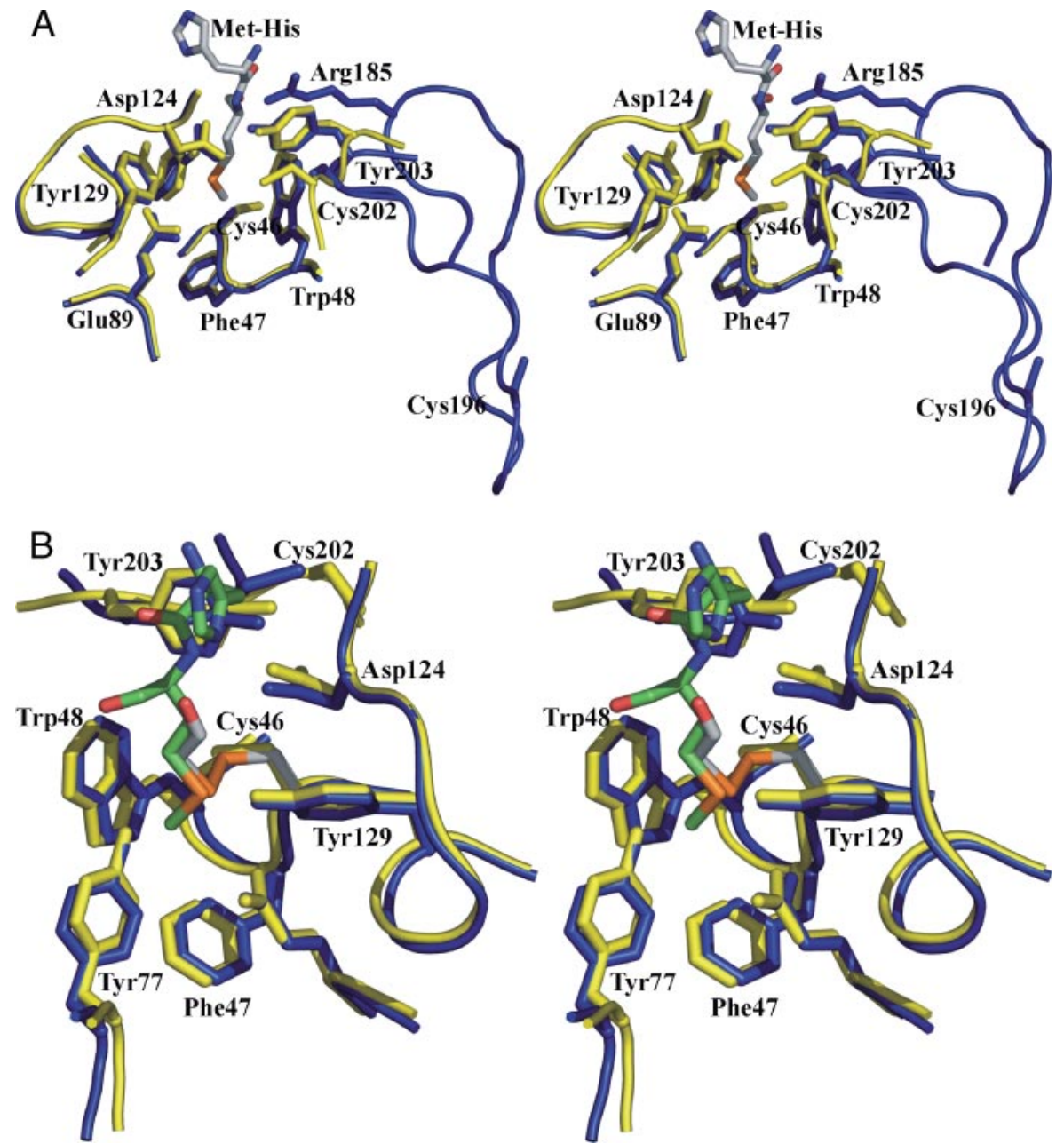

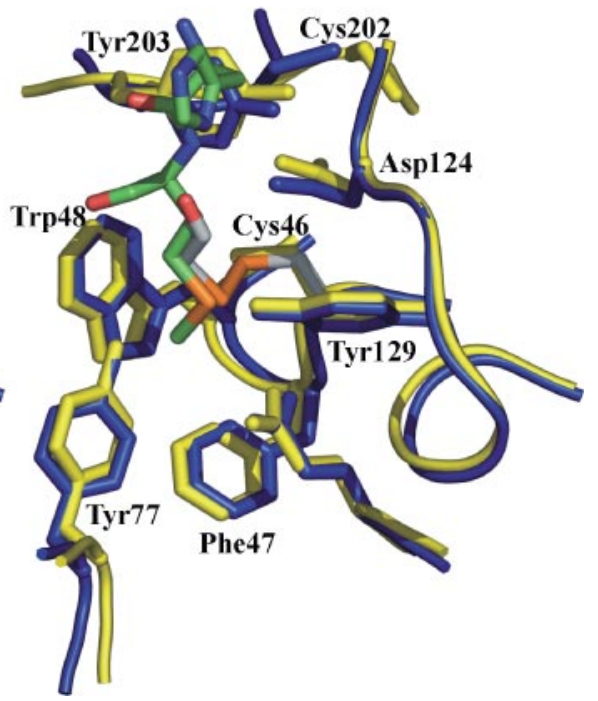

FIGURE 6. Stereoviews showing a superimposition of the pMsrA (blue) and M. tuberculosis MsrA (yellow) models in the active site region. $A$, the $C-\alpha$ trace of the pMsrA structure is shown in coil mode. The methionine residue observed in the $M$. tuberculosis active site is shown in stick mode. $B$, detailed view that emphasizes the comparable stabilization of the $\beta$-mercaptoethanol molecule bound to the catalytic cysteine in the pMsrA model and the methionine residue observed in the catalytic site of the M. tuberculosis enzyme.

to that observed in M. tuberculosis MsrA between the side chain of the equivalent aspartate residue $\left(\mathrm{Asp}^{87}\right)$ and the amide group of the methionine main chain (14) (Fig. 6). Modeling of a peptidic methionine sulfoxide bound in the active site of pMsrA, based on the M. tuberculosis model, shows the following: 1) the invariant Phe ${ }^{47}$ and $\operatorname{Trp}^{48}$ of the GCFW active-site consensus sequence form a hydrophobic pocket in which the $\epsilon$-methyl group of MetSO can bind as already observed in all the three-dimensional structures of MsrAs determined so far; 2) the oxygen of the sulfoxide is situated at hydrogen bonding distances of $\mathrm{Tyr}^{77}, \mathrm{Glu}^{89}$, and $\mathrm{Tyr}^{129}$ (Fig. 6); and 3) the location of Asp $^{124}$ is suitable for its involvement in the stabilization of the main chain of the substrate. In M. tuberculosis MsrA, the side chain of the equivalent aspartate residue $\left(\mathrm{Asp}^{87}\right)$ points toward the side chain of $\mathrm{Tyr}^{152}$, which belongs to the GYTC sequence (the ending cysteine residue being CysB). The aromatic ring of this tyrosine residue is proposed to stack the peptidic bond between the methionine sulfoxide of the substrate and its upstream residue, through a $\pi-\pi$ interaction (14). In the B. taurus MsrA structure, a similar interaction is observed between structurally equivalent aspartate and tyrosine resi- dues. In this case, the tyrosine residue $\left(\mathrm{Tyr}^{217}\right)$ belongs to the more frequent GYC sequence (see under "Genome and Sequence Analysis"). As mentioned earlier, the GYC or GYXC string is absent from the plant MsrA sequences. However, in the latter, a stabilizing tyrosine residue is still observed, because $\mathrm{Tyr}^{203}$ of pMsrA is structurally equivalent to $\mathrm{Tyr}^{217}$ of the B. taurus structure or to $\mathrm{Tyr}^{152}$ of the M. tuberculosis enzyme. The new conformation of the $\mathrm{C}$-terminal end observed in the plant structure allows the poplar main chain of only three residues, ${ }^{202} \mathrm{CYG}^{204}$, to run at roughly the same position than the main chains of the other two structures, but in an antiparallel way. The reverse direction of the superposed polypeptidic chains thus remarkably places the C- $\alpha$ atoms of the CYG string of residues in the poplar enzyme at roughly the same location as the $\mathrm{C}-\alpha$ atoms of the ${ }^{218} \mathrm{CYG}^{216}$ residues of $B$. taurus MsrA or as the $C-\alpha$ atoms of the ${ }^{153} \mathrm{TYG}^{151}$ residues in the $M$. tuberculosis MsrA structure. In each case, it allows the central tyrosine residue to exactly maintain the same position and orientation of its side chain. The side chain of $\mathrm{Tyr}^{202}$, conserved in plants through a C-terminal CYG sequence, is thus proposed to act as $\mathrm{Tyr}^{152}$ in $M$. tuberculosis MsrA, conserved in a GYXC or GYC sequence in a large subset of MsrAs.

The conformation of the $\mathrm{C}$-terminal end observed in the pMsrA structure also suggests that the extended side chain of $\operatorname{Arg}^{185}$ participates in substrate binding through a hydrogen bond with the main chain of the methionine sulfoxide. The substrate could then be maintained within the active site by interactions on both sides of its main chain. However, the position of this arginine residue is shifted by one amino acid in some plant sequences, and a substitution for an asparagine residue is observed in Fragaria anannassa MsrA. Whether these differences are compatible with the proposed role of $\mathrm{Arg}^{185}$ remains to be assessed.

Position of the Cysteine Residues-Among the five conserved cysteines, Cys ${ }^{46}$ (CysA) lies at the bottom of the active site. A comparative analysis with other MsrA structures shows that its bonding to the $\beta$-mercaptoethanol molecule reasonably mimics part of the transient tetrahedral intermediate formed after the CysA attack onto the substrate (see Fig. 6). Cys $^{196}$ (CysB) is found far from the active site, near the C-terminal end of $\alpha$-helix H1, in the portion of the C-terminal coil that runs antiparallel to this $\alpha$-helix. This location of $\mathrm{Cys}^{196}$ places its sulfur 
atom at a distance of $18 \AA$ from the S- $\gamma$ atom of CysA. Cys ${ }^{202}$ (CysC), as part of the C-terminal CYG sequence of plant MsrAs, is found near the active site, with its $\mathrm{C}-\alpha$ atom being in a position close to the C- $\alpha$ atom of the B. taurus MsrA CysB $\left(\mathrm{Cys}^{218}\right)$. However, the torsion angles of the polypeptide chain direct the CysC side chain of the poplar enzyme opposite to that of B. taurus MsrA. Cys ${ }^{202}$ makes hydrophobic contacts with the aromatic side chain of $\mathrm{Tyr}^{203}$ and elongates its bonded $\beta$-mercaptoethanol parallel to the $\mathrm{Asp}^{124}-\mathrm{Tyr}^{203}$ pair of residues. The sulfur-sulfur distance between $\mathrm{Cys}^{202}$ and $\mathrm{Cys}^{196}$ is $15.8 \AA$, whereas it is only $7.1 \AA$ with Cys ${ }^{46}$. Rotations of the $\mathrm{Cys}^{46}$ and $\mathrm{Cys}^{202}$ side chains bring their sulfur atoms to a minimum distance of $3.3 \AA$ that could allow the formation of the disulfide bond. However, the required movement of $\mathrm{Cys}^{202}$ would be slightly hindered by the $\mathrm{Tyr}^{203}$ side chain, maintained in position through an interaction with $\mathrm{Asp}^{124}$. It thus necessitates that small conformational changes occur during the first step of the regeneration process of the enzyme. On the contrary, large movements are necessary to approach Cys ${ }^{196}$ toward Cys ${ }^{46}$. Such a requirement seems to be a common property of all MsrAs that involve three cysteine residues in their catalytic mechanism. Indeed, in the B. taurus MsrA structure (13), the $\mathrm{C}-\alpha$ atom of $\mathrm{CysB}\left(\mathrm{Cys}^{218}\right)$ is found at $7.1 \AA$ of the $\mathrm{C}-\alpha$ atom of CysA $\left(\mathrm{Cys}^{72}\right)$, but the $\mathrm{CysB}$ side chain points outside the active site and cannot be reoriented toward CysA by simply modifying its side chain torsion angle. Furthermore, $\mathrm{CysC}\left(\mathrm{Cys}^{227}\right)$ is situated at $21 \AA$ from CysB. In the E. coli MsrA structure (12), the $\mathrm{C} \alpha-\mathrm{C} \alpha$ atom distance for CysA $\left(\mathrm{Cys}^{51}\right)$ and $\mathrm{CysB}\left(\mathrm{Cys}^{198}\right)$ is 11 $\AA$, whereas it is $20 \AA$ between $\mathrm{CysB}$ and $\mathrm{CysC}\left(\mathrm{Cys} 2^{06}\right)$. However, the disulfide bond is supposed to form once the product, i.e. the reduced methionine, is released, concomitantly with the formation of the sulfenic acid on CysA. These events might modify the enzyme conformation, especially at the C-terminal end, in order to approach CysB or CysC (for the poplar enzyme) closer to the sulfenic acid form on CysA. Only three-dimensional structures of an oxidized state of MsrA would bring an answer that cannot be deduced from the current structures that represent either a Michaelis-like complex or a transition state preceding formation of the sulfenic intermediate. Concerning poplar pMsrA, the structure determination of the oxidized forms of the enzyme would help to understand the nature of the conformational changes that occur at the different steps of the catalytic mechanism. However, to date, attempts to crystallize such forms of the enzyme have failed.

Conclusion-The biochemical studies indicate, as expected, that the enzyme is able to reduce the $S$ enantiomer of MetSO using Trx as a reductant. Out of a total of five cysteines, three are involved in the catalytic mechanism, the catalytic $\mathrm{Cys}^{46}$, on which the sulfenic intermediate is formed, and two recycling cysteines ( $\mathrm{Cys}^{196}$ and $\mathrm{Cys}^{202}$ ), which are involved in the Trx-dependent recycling process and are located in a highly conserved motif specific to plant enzymes. Inspection of the three-dimensional structure of the poplar pMsrA suggests a Trx-dependent recycling process for this plant MsrA different from the one described for the E. coli and B. taurus enzymes. The sulfenic acid formed on $\mathrm{Cys}^{46}$ after MetSO reduction would be attacked by the more $\mathrm{C}$-terminal $\mathrm{Cys}^{202}$ (CysC), leading to formation of a $\mathrm{Cys}^{46}-\mathrm{Cys}^{202}$ disulfide bond. Then, Cys ${ }^{196}$ (CysB) would attack the disulfide bond to form a $\mathrm{Cys}^{202}$ - $\mathrm{Cys}^{196}$ bond, which is finally reduced by Trx. The conformational changes needed to place $\mathrm{Cys}^{202}$ close to the active site and especially close to $\mathrm{Cys}^{46}$ are small. In addition, $\mathrm{Cys}^{202}$ is included in a CYG motif, which is conserved in all plant MsrAs. The tyrosine residue corresponds to the one described to be involved in substrate binding in bacterial and B. taurus MsrAs. In these MsrAs, the tyrosine residue belongs to a similar motif as found for $\mathrm{pMsrA}$ but with the first $\mathrm{C}$-terminal cysteine instead of the last $\mathrm{C}$-terminal cysteine.

Acknowledgments-We are very grateful to Jean-Luc Ferrer and Pierre Legrand at the ESRF (beamline BM30A) for giving us the opportunity to perform data collection and for their kind help during this experiment.

\section{REFERENCES}

1. Imlay, J. A., and Linn, S. (1986) Science 240, 1302-1309

2. Berlett, B., and Stadman, E. (1997) J. Biol. Chem. 272, 20313-20316

3. Vogt, W. (1995) Free Radic. Biol. Med. 18, 93-105

4. Brot, N., Weissbach, L., Werth, J., and Weissbach, H. (1981) Biochemistry 78, 2155-2158

5. Grimaud, R., Ezraty, B., Mitchell, J. K., Lafitte, D., Briand, C., Derrick, P. J., and Barras, F. (2001) J. Biol. Chem. 276, 48915-48920

6. Gao, J., Yin, D. H., Yao, Y., Sun, H., Qin, Z., Schoneich, C., Williams, T. D., and Squier, T. C. (1998) Biophys. J. 74, 1115-1134

7. Harndahl, U., Kokke, B. P., Gustavsson, N., Linse, S., Berggren, K., Tjerneld, F., Boelens, W. C., and Sundby, C. (2001) Biochim. Biophys. Acta 1545, 227-237

8. Taggart, C., Cervantes-Laurean, D., Kim, G., McElvaney, N. G., Wehr, N., Moss, J., and Levine, R. L. (2000) J. Biol. Chem. 275, 27258-27265

9. Khor, H. K., Fisher, M., and Schöneich, C. (2004) J. Biol. Chem. 279, 19486-19493

10. Sun, H., Gao, J., Ferrington, D. A., Biesiada, H., Williams, T. D., and Squier, T. C. (1999) Biochemistry 38, 105-112

11. Levine, R. L., Mosoni, L., Berlett, B. S., and Stadman, E. R. (1996) Proc. Natl. Acad. Sci. U. S. A. 93, 15036-15040

12. Tete-Favier, F., Cobessi, D., Boschi-Muller, S., Azza, S., Branlant, G., and Aubry, A. (2000) Structure 8, 1167-1178

13. Lowther, W. T., Brot, N., Weissbach, H., and Matthews, B. W. (2000) Biochemistry 39, 13307-13312

14. Taylor, A. B., Benglis, D. M., Jr., Dhandayuthapani, S., and Hart, P. J. (2003) J. Bacteriol. 185, 4119-4126

15. Lowther, W. T., Weissbach, H., Etienne, F., Brot, N., and Matthews, B. W. (2002) Nat. Struct. Biol. 9, 348-352

16. Kauffmann, B., Aubry, A., and Favier, F. (2005) Biochim. Biophys. Acta 1703, 249-260

17. Boschi-Muller, S., Olry, A., Antoine, M., and Branlant, G. (2005) Biochim. Biophys. Acta 1703, 231-238

18. Boschi-Muller, S., Azza, S., Sanglier-Cianferani, S., Talfournier, F., Van Dorsselear, A., and Branlant, G. (2000) J. Biol. Chem. 275, 35908-35913

19. Kumar, R. A., Koc, A., Cerny, R. L., and Gladyshev, V. N. (2002) J. Biol. Chem. 277, 37527-37535

20. Boschi-Muller, S., Azza, S., and Branlant, G. (2001) Protein Sci. 10, 2272-2279

21. Sadanandom, A., Poghosyan, Z., Fairbairn, D. J., and Murphy, D. J. (2000) Plant Physiol. 123, 255-264.

22. Romero, H. M., Berlett, B. S., Jensen, P. J., Pell, E. J., and Tien, M. (2004) Plant Physiol. 136, 3784-3794.

23. Vieira Dos Santos, C., Cuine, S., Rouhier, N., and Rey, P. (2005) Plant Physiol. 138, 909-922

24. Gustavsson, N., Kokke, B. P., Harndahl, U., Silow, M., Bechtold, U., Poghosyan, Z., Murphy, D., Boelens, W. C., and Sundby, C. (2002) Plant J. 29, 545-553 


\section{Biochemical and Structural Characterization of Plant MsrAs}

25. Bechtold, U., Murphy, D. J., and Mullineaux, P. M. (2004) Plant Cell 16, 908-919

26. In, O., Berberich, T., Romdhane, S., and Feierabend, J. (2005) Planta 220, 941-950

27. Sadanandom, A., Piffanelli, P., Knott, T., Robinson, C., Sharpe, A., Lydiate, D., Murphy, D., and Fairbairn, D. J. (1996) Plant J. 10, 235-242

28. Jacquot, J. P., Stein, M., Suzuki, A., Liottet, S., Sandoz, G., and MiginiacMaslow, M. (1997) FEBS Lett. 400, 293-296

29. Schenk, P. M., Baumann, S., Mattes, R., and Steinbiss, H. H. (1995) BioTechniques 19, 196-200

30. Otwinowski, Z., and Minor, W. (1997) Methods Enzymol. 276, 307-326

31. Vagin, A., and Teplyakov, A. (1997) J. Appl. Crystallogr. 30, 1022-1025

32. Perrakis, A., Morris, J. R., and Lamzin, V. S. (1999) Nat. Struct. Biol. 6,
$458-463$

33. Brünger, A. T., Adams, P. D., Clore, G. M., DeLano, W. L., Gros, P., Grosse-Kunsteleve, R. W., Jiang, J. S., Kuszewski, J., Nilges, M., Pannu, N. S., Read, R. J., Rice, L. M., Simonson, T., and Warren, G. L. (1998) Acta Crystallogr. Sect. D Biol. Crystallogr. 54, 905-921

34. Jacquot, J. P., Rivera-Madrid, R., Marinho, P., Kollarova, M., Le Marechal, P., Miginiac-Maslow, M., and Meyer, Y. (1994) J. Mol. Biol. 235, 1357-1363

35. Behm, M., and Jacquot, J. P. (2000) Plant Physiol. Biochem. 38, 363-369

36. Olry, A., Boschi-Muller, S., Marraud, M., Sanglier-Cianferani, S., Van Dorsselear, A., and Branlant, G. (2002) J. Biol. Chem. 277, 12016-12022

37. Antoine, M., Boschi-Muller, S., and Branlant, G. (2003) J. Biol. Chem. 278, $45352-45357$ 